\title{
THE CAUCHY-POISSON WAVES IN AN INVISCID ROTATING STRATIFIED LIQUID
}

\author{
Lokenath Debnath and Uma B. Guha \\ Department of Mathematics \\ University of Central Florida \\ Orlando, FL 32816 \\ and \\ Manjusri Basu \\ Department of Mathematics \\ Kalyani University \\ Kalyani, West Bengal, India
}

\begin{abstract}
Based upon the Boussinesq approximation, an initial value investigation is made of the axisymmetric free surface flows generated in an inviscid rotating stratified liquid of infinite depth by the prescribed free surface disturbance. The asymptotic analysis of the integral solution is carried out by the stationary phase method to describe the solution for large time and large distance from the source of the disturbance. The asymptotic solution is found to consist of the classical free surface gravity waves and the internal-inertial waves.
\end{abstract}

\section{AMS Subject Classification:} 76B15,76D33,76C10.

Key Words: Surface waves, internal waves, inertial waves, asymptotic solution, waves in oceans.

\footnotetext{
${ }^{0}$ Received: March 1989; Revised: September 1989
} 


\section{INTRODUCTION.}

Recently Debnath and Guha (1989) have studied the Cauchy-Poisson problem in an inviscid stratified liquid. This problem in a rotating liquid is of special interest in geophysical fluid dynamics. So the main purpose of this paper is to generalize the Cauchy-Poisson problem in a uniformly rotating inviscid stratified liquid of infinite depth.

This paper is concerned with the initial value investigation of the axisymmetric free surface flows generated in an inviscid rotating stratified liquid of infinite depth by the prescribed surface disturbance. Based upon the Boussinesq approximation, the problem is solved by the joint use of the Laplace and Hankel transforms. The formal integral solution for the free surface elevation is obtained. Special attention is given to the governing dispersion relations in the rotating stratified liquid with or without free surface curvature. The limiting cases of the general dispersion relation are discussed as $N$ or $2 \Omega$ tends to zero. The asymptotic analysis of the integral solution is carried out by the stationary phase approximation to determine the nature of the Cauchy-Poisson waves for large time and distance from the source of the disturbance. The asymptotic solution is found to consist of the free surface gravity waves and internal-inertial waves. The effects of both stratification and rotation are examined. The results of this paper reduce to those of Debnath and Guha (1989) in the absence of rotation $(\Omega \rightarrow 0)$.

\section{MATHEMATICAL FORMULATION.}

We consider the axisymmetric Cauchy-Poisson problem in an inviscid incompressible rotating stratified liquid of infinite depth. We use the cylindrical coordinates $(r, \theta, z)$ and consider a semi-infinite body of liquid bounded by $0 \leq r<\infty,-\infty<z \leq z_{0}(r)$. The liquid is subjected to a uniform rotation with angular velocity $\Omega$ about the vertical axis $r=0$ so that the equation of the paraboloidal free surface with $2 l$ as the latus rectum is given by

$$
z=z_{0}(r)=\frac{r^{2}}{2 l} .
$$

We assume that the disturbed free surface is given by

$$
z=z_{0}(r)+\eta(r, t)
$$


due to the superimposed initial elevation

$$
z-z_{0}=\eta_{0}(r)=a \frac{\delta(r)}{r} \text { at } t=0
$$

where $2 \pi a$ is the displaced volume associated with $\eta_{0}$ and $\delta(r)$ is the Dirac function.

In the rotating frame of reference, the unsteady motion of the liquid is governed by the Boussinesq equations (Greenspan, 1968; Debnath, 1974)

$$
\rho\left(\frac{\delta \bar{u}}{\delta t}+(\bar{u} \cdot \nabla \bar{u})\right)+\rho(2 \bar{\Omega} \times \bar{u})=-\nabla p+\rho \bar{g}
$$

where $\rho$ is the density, $\bar{u}=(u, v, w)$ is the velocity vector, $\bar{\Omega}=\bar{k} \Omega$ is the rotation vector, $\bar{k}$ is the unit vector parallel to the $z$-axis, $p$ is the modified pressure including centrifugal acceleration, and $\bar{g}=(0,0, g)$ is the gravitational acceleration.

The equation of incompressibility of the liquid is

$$
\frac{\delta \rho}{\delta t}+\bar{u} \cdot \nabla \rho=0
$$

The continuity equation in vector notation is

$$
\nabla \cdot \bar{u}=0 .
$$

If $p$ and $\rho$ are expanded about $p_{0}$ and $\rho_{0}$ in a reference state of hydrostatic equilibrium then

$$
\begin{aligned}
\nabla p_{0} & =g \rho_{0} \\
p & =p_{0}(z)+p^{\prime}(z, t) \\
\rho & =\rho_{0}(z)+\rho^{\prime}(z, t) .
\end{aligned}
$$

when $p^{\prime}$ and $\rho^{\prime}$ are the perturbed quantities. We further assume that the Rossby number is very small and $\eta_{0}$ is sufficiently small to justify the linearization of both the equations of motion and the free surface conditions. The present problem will be studied under the Boussinesq approximation which states that the density variation involved in the inertia and the Coriolis terms can be neglected but it must be retained in the buoyancy force term in (4). The density field of the undisturbed liquid is supposed to be of the form $\rho_{0}=\rho_{00} \exp (-\beta z)$, where $\beta$ is a positive constant. The Brunt Vaisala frequency $N$ given by

$$
N=\left(-\frac{g}{\rho_{0}} \frac{d \rho_{0}}{d z}\right)^{1 / 2}
$$


is real and positive when the mean distribution is stable $\left(\frac{d \rho 0}{d z}<0\right)$ and it remains constant $(N=\sqrt{g \beta})$ throughout the flow field.

In view of the above assumptions combined with the acceleration potential $\chi=\left(p^{\prime} / \rho_{0}\right)+g\left(z-z_{0}\right)$, the governing equations assume the form

$$
\frac{\delta}{\delta t}(u, v, w)+2 \Omega(-v, u, 0)=-\left(\frac{\delta}{\delta r}, 0, \frac{\delta}{\delta z}\right) \chi-\left(0,0, \frac{g \rho^{\prime}}{\rho_{0}}\right),
$$

$$
\begin{aligned}
\frac{\delta \rho^{\prime}}{\delta t}+w \frac{d \rho_{0}}{d z} & =0 \\
\frac{\delta u}{\delta r}+\frac{u}{r}+\frac{\delta w}{\delta z} & =0
\end{aligned}
$$

The free surface conditions are

$$
\chi=g \eta, \quad w=\eta_{t}+u z_{0}^{\prime}(r) \text { on } z=z_{0} .
$$

The bottom boundary condition is

$$
\frac{\delta \chi}{\delta z} \rightarrow 0 \text { as } z \rightarrow-\infty \text {. }
$$

The wave motion is generated in the liquid by the action of the initial surface elevation at $t=0$ so that the initial conditions are

$$
\bar{u}=\chi=0, \quad \eta(r, t)=\eta_{0}(r) \text { at } t=0 .
$$

\section{THE INTEGRAL SOLUTION AND THE DISPERSION RELATION.}

We first transform the initial value problem (11)-(16) to a boundary value problem by using the Laplace transformation with respect to $t$ (see Myint-U and Debnath, 1987). We then eliminate the transform functions $\tilde{u}, \tilde{v}, \tilde{w}$, and $\tilde{p}^{\prime}$ to derive a single equation for $\tilde{\chi}$ and the transformed free surface and boundary conditions as

$$
\begin{gathered}
\tilde{\chi}_{r r}+\frac{1}{r} \tilde{\chi}_{r}+\lambda^{2} \tilde{\chi}_{z z}=0 \\
\lambda^{2}=\frac{s^{2}+4 \Omega^{2}}{s^{2}+N^{2}}
\end{gathered}
$$




$$
\begin{gathered}
\frac{s^{2}}{g} \bar{\chi}+\frac{s^{2}}{s^{2}+N^{2}} \tilde{\chi}_{z}-\frac{s^{2}}{s^{2}+4 \Omega^{2}} \tilde{\chi}_{r} z_{0}^{\prime}(r)=s \eta_{0}(r) \\
\tilde{\chi}_{z} \rightarrow 0 \text { as } z \rightarrow-\infty .
\end{gathered}
$$

It is noted that (17) is Laplace's equation in the coordinates $r$ and $z / \lambda$, and $z=z_{0}=r^{2} / 2 l$ is a paraboloid of revolution. It is convenient to introduce parabolic coordinates $\xi$ and $\zeta$ in the $(r, z / \lambda)$-space. We normalize these coordinates such that $\xi=r$ and $\zeta=1$ on the free surface $z=z_{0}(r)$, and introduce the transformation in the form

$$
\begin{aligned}
r=\xi \zeta & 0 \leq \xi<\infty, 1 \leq \zeta<\infty \\
z-z_{0}=\frac{1}{2}\left[\frac{\xi^{2}}{l}+\lambda^{2}\left(1-\zeta^{2}\right)\right] & 0 \leq \xi<\infty, 1 \leq \zeta<\infty
\end{aligned}
$$

The differential metric is given by

$$
(d r)^{2}+\left(\frac{d z}{\lambda}\right)^{2}=\left[\frac{\xi^{2}}{\lambda l}+\lambda l \zeta^{2}\right]\left[\frac{(d \xi)^{2}}{\lambda l}+\lambda l(d \zeta)^{2}\right] .
$$

In view of these transformations, the system (17)-(19) reduces to

$$
\begin{aligned}
& (\lambda l)^{2} \xi^{-1}\left(\xi \tilde{\chi}_{\xi}\right)_{\xi}+\zeta^{-1}\left(\zeta \tilde{\chi}_{\zeta}\right)_{\zeta}=0 \\
& \frac{s^{2}}{g} \tilde{\chi}-\left(\lambda^{2} l\right)^{-1} \tilde{\chi}_{\zeta}=s \eta_{0}(\xi), \quad \zeta=1 .
\end{aligned}
$$

It is noted that $\xi$ and $\zeta$ are orthogonal coordinates in the $(r, z / \lambda)$ space and $\xi$ has a dimension of length and $\zeta$ is dimensionless. Both $\xi$ and $\zeta$ are real only for real values of $\lambda^{2}$. Equation (24) is hyperbolic or elliptic according to whether $\lambda^{2}<0$ or $>0$.

We next assume $\tilde{\chi}$ is bounded as $\xi \rightarrow 0, \xi \rightarrow \infty$, and $\zeta \rightarrow \infty$ and seek a particular solution of the system (24)-(25) in the form

$$
\tilde{\chi}=J_{0}(k \xi) K_{0}(\lambda k l \zeta), \quad \operatorname{Re}(\lambda)>0
$$

where $J_{0}(x)$ is the Bessel function of the first kind of order zero and $K_{0}(x)$ is the modified Bessel function of the second kind.

Introducing the joint Laplace and Hankel transform (see Myint- $U$ and Debnath, 1987)

$$
\overline{\tilde{\eta}}(k, s)=\int_{0}^{\infty} e^{-s t} d t \int_{0}^{\infty} r J_{0}(k r) \eta(r, t) d r
$$


we obtain the integral solutions of $(24)-(25)$ with (3) so that $\bar{\eta}_{0}(k)=a$ in the form

$$
\chi(r, z, t)=\frac{a g}{2 \pi i} \int_{c-i \infty}^{c+i \infty} \frac{s e^{s t}}{s^{2}+N^{2}} d s \int_{0}^{\infty} \frac{k J_{0}(k r) P(\lambda k l \zeta)}{s^{2}+N^{2}+\frac{g k}{\lambda} \psi(\lambda k l)} d k
$$

$$
\begin{aligned}
\eta(r, t) & =\lim _{\zeta \rightarrow 1+} \frac{a}{2 \pi i} \int_{c-i \infty}^{c+i \infty} \frac{s^{2}+N^{2}}{s} e^{s t} d s \int_{0}^{\infty} \frac{k J_{0}(k r) P(\lambda k l \zeta)}{s^{2}+N^{2}+\frac{g k}{\lambda} \psi(\lambda k l)} d k \\
& =\frac{a}{2 \pi i} \int_{c-i \infty}^{c+i \infty} \frac{s^{2}+N^{2}}{s} e^{s t} d s \int_{0}^{\infty} \frac{k J_{0}(k r) Q(\lambda k l \zeta)}{s^{2}+N^{2}+\frac{g k}{\lambda} \psi(\lambda k l)} d k
\end{aligned}
$$

where

$$
\begin{gathered}
P(\kappa \zeta)=\frac{K_{0}(\kappa \zeta)}{K_{0}(\kappa)} \quad \kappa=\lambda k \\
\psi(\kappa)=\frac{K_{1}(\kappa)}{K_{0}(\kappa)}=\frac{i H_{1}^{(1)}(i \kappa)}{H_{0}^{(1)}(i \kappa)} \sim 1+\frac{1}{2 \kappa}+O\left(\frac{1}{\kappa^{2}}\right) \text { as } \quad \kappa \rightarrow \infty
\end{gathered}
$$

and $Q$ is the limit of $P$ as $\zeta \rightarrow 1+$ after the double integral with respect to $s$ and $k$ has been evaluated.

We also note that

$$
P(\kappa \zeta) \sim \exp [-\kappa(\zeta-1)] \text { as }|\kappa| \rightarrow \infty, \operatorname{Re}(\kappa)>0, \zeta>1 .
$$

A careful inspection of $(30)$ reveals that the $s$-integral has three poles at $s=0$ and $s= \pm i \omega$, and the dispersion relation is obtained by replacing $s$ by $\pm i \omega$ in the expression for $\lambda$ and then equating the denominator of (30) to zero so that

$$
\begin{aligned}
\omega^{2} & =N^{2}+\frac{g k}{\lambda} \psi(\lambda k l) \\
\lambda^{2} & =\frac{\omega^{2}-4 \Omega^{2}}{\omega^{2}-N^{2}} .
\end{aligned}
$$

In order to simplify this result, we observe that equilibrium between the constant gravitational field and the centrifugal acceleration at $z=z_{0}$ leads to $l=g / \Omega^{2}$ so that a dimensionless parameter $\alpha=\Omega^{2} l / g$ can be introduced. Clearly $\alpha \rightarrow \infty(l \rightarrow \infty)$ corresponds to the horizontal free surface of the liquid. In this limit, the free-surface curvature of the liquid will be absent and (32) is used to simplify (34) in the form

$$
\left(\omega^{2}-N^{2}\right)^{2} \lambda^{2}=g^{2} k^{2} .
$$


This is the dispersion relation in a uniformly rotating stratified liquid. In the absence of rotation $(\Omega=0)$, the dispersion relation agrees with Debnath and Guha (1989). On the other hand, when there is no stratification $(N=0),(36)$ reduces to the corresponding dispersion relation for a rotating liquid. Finally, in the limits $2 \Omega \rightarrow 0$ and $N \rightarrow 0$, the dispersion relation is approximately equal to

$$
\omega^{2} \sim \frac{1}{2}\left(N^{2}+4 \Omega^{2}\right)+g k .
$$

This reduces to the famous dispersion relation $\left(\omega^{2}=g k\right)$ in deep water in a non-rotating, non-stratified case.

\section{ASYMPTOTIC REPRESENTATION OF THE FREE SURFACE ELEVATION.}

In order to determine the ultimate nature of the waves in a rotating stratified liquid, the asymptotic behavior of the solution for sufficiently large time is of special interest. We next introduce the non-dimensional elevation $\eta^{*}=\left(r^{2} / a\right) \eta(r, t)$ where $\eta(r, t)$ is given by (30), and write

$$
\begin{aligned}
\eta^{*} & =\frac{r^{2}}{a} \frac{1}{2 \pi i} \int_{c-i \infty}^{c+i \infty} \frac{s^{2}+N^{2}}{s} e^{s t} d s \int_{0}^{\infty} \frac{k J_{0}(k r) Q(\lambda k l \zeta)}{s^{2}+N^{2}+\frac{g k}{\lambda} \psi(\lambda k l)} d k \\
& =\eta_{1}^{*}+\eta_{2}^{*}
\end{aligned}
$$

where $\eta_{1}^{*}$ and $\eta_{2}^{*}$ are made up of the polar and branch-point contributions respectively.

Making the change of variable $k r=u / \lambda$ and introducing the non-dimensional parameters $\mu=g t^{2} / r, \tau=\Omega t, \theta=r / l, \alpha \theta=\Omega^{2} r / g, \beta \theta=N^{2} r / g$, we rewrite (38) and then apply the Cauchy theorem of residue to obtain the polar contribution as

$$
\begin{aligned}
\eta_{1}^{*}(r, t)= & r^{2} \int_{0}^{\infty}\left(1-\frac{N^{2}}{\omega^{2}}\right) \cos (\omega t) J_{0}(k r) Q k d k \\
& +r^{2} \Omega^{2} \int_{0}^{\infty} \frac{k J_{0}(k r)}{\omega^{2}} d k .
\end{aligned}
$$

We next replace $J_{0}(k r)$ by its asymptotic value for large $k r$, and then apply the method of stationary phase to evaluate $\eta_{1}^{*}$ for large $\mu$. It turns out that

$$
\eta_{1}^{*} \sim \frac{\frac{\mu}{2}-\beta-\frac{3 \alpha}{2}}{\frac{\mu}{4}-\beta-\frac{\alpha}{2}}\left(\frac{\mu}{2}\right)^{-1 / 2} \cos \left(\frac{g t^{2}}{4 r}-2 \beta-\frac{\alpha}{2}\right) \text { as } \mu \rightarrow \infty .
$$


Similarly, $\eta_{2}^{*}$ can also be evaluated asymptotically to obtain

$$
\begin{aligned}
\eta_{2}^{*} \sim & \frac{2 N r}{\pi g t} J_{1}(N t)-\frac{4 \Omega r}{\pi g t} J_{1}(2 \Omega t) \quad \text { as } N t, \Omega t \rightarrow \infty \\
\sim & \frac{2 \beta \theta}{\sqrt{\pi}}(N t)^{-3 / 2} \sin \left(N t-\frac{\pi}{4}\right) \\
& -\frac{8 \alpha \theta}{\sqrt{\pi}}(2 \Omega t)^{-3 / 2} \sin \left(2 \Omega t-\frac{\pi}{4}\right) \quad \text { as } N t, \Omega t \rightarrow \infty .
\end{aligned}
$$

A simple combination of (41) and (42) gives an asymptotic solution for $\eta(r, t)$ which consists of three distinct terms representing waves. The term (41) corresponds to surface waves which are qualitatively similar to those in the classical Cauchy-Poisson waves in an inviscid, non-rotating, non-stratified liquid. However, the amplitude of those waves is modified by both rotation and stratification. But the main effect of rotation and stratification is the phase shift by an amount $2 \beta+\alpha / 2$ in the asympototic wavetrains. The terms in (42) correspond to waves of frequency $N$ and $2 \Omega$ and the amplitudes of these waves decay to zero as $N t \rightarrow \infty$ and $\Omega t \rightarrow \infty$. These are not surface waves and their existence is entirely due to rotation and density-stratification. They have no antecedents in a non-rotating and non-stratified inviscid liquid. In the absence of rotation $(2 \Omega=0)$, this analysis is in perfect agreement with that of Debnath and Guha (1989) in an inviscid stratified liquid.

Acknowledgement: The authors express their grateful thanks to the referee for suggesting some improvements in the paper.

\section{REFERENCES.}

1. Debnath, L. and Guha, U., 1989, "The Cauchy-Poisson Problem in an Inviscid Stratified Liquid," Appl. Math. Letters, Vol. 2, No. 4, $337-440$.

2. Greenspan, H.P., The Theory of Rotating Liquids, Cambridge University Press, 1968.

3. Debnath, L., 1974, "On Forced Oscillations in a Rotating Stratified Liquid," Tellus, 26:652-662.

4. Myint-U, T. and Debnath, L., Partial Differential Equations for Scientists and Engineers, Third Edition, North Holland, 1987.

5. Lamb, H., Hydrodynamics, Cambridge University Press, 1932. 


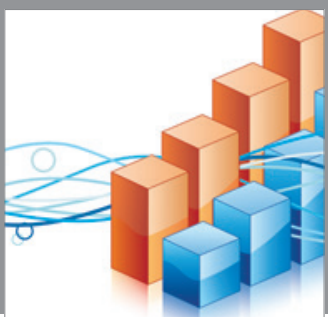

Advances in

Operations Research

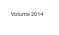

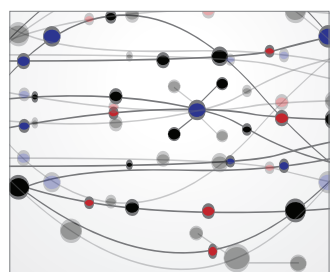

\section{The Scientific} World Journal
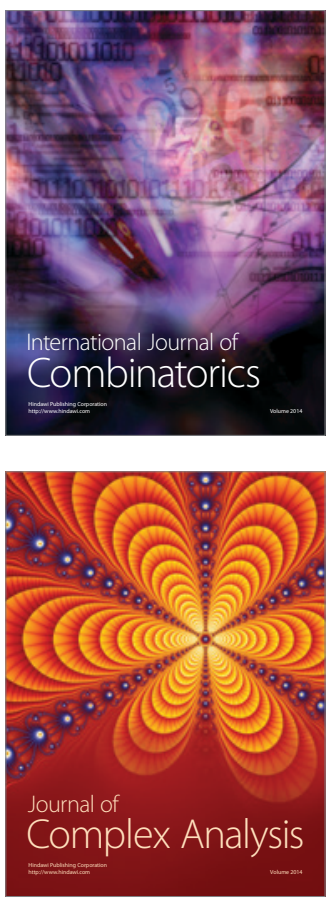

International Journal of

Mathematics and

Mathematical

Sciences
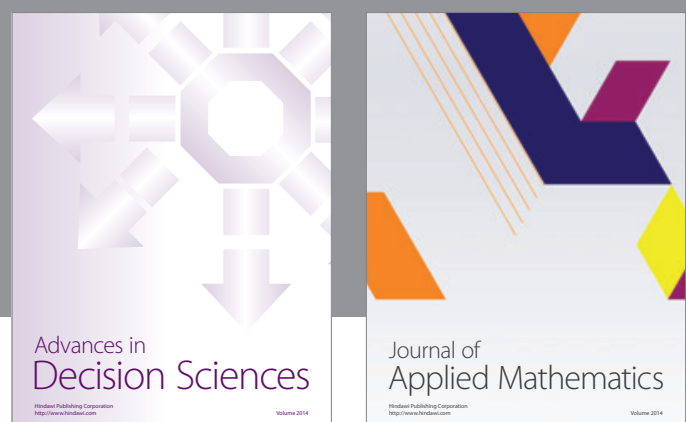

Journal of

Applied Mathematics
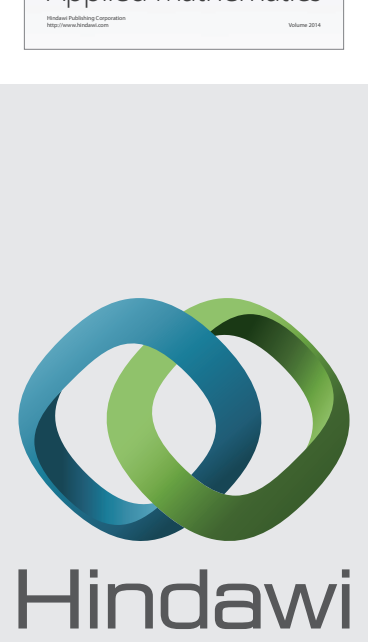

Submit your manuscripts at http://www.hindawi.com
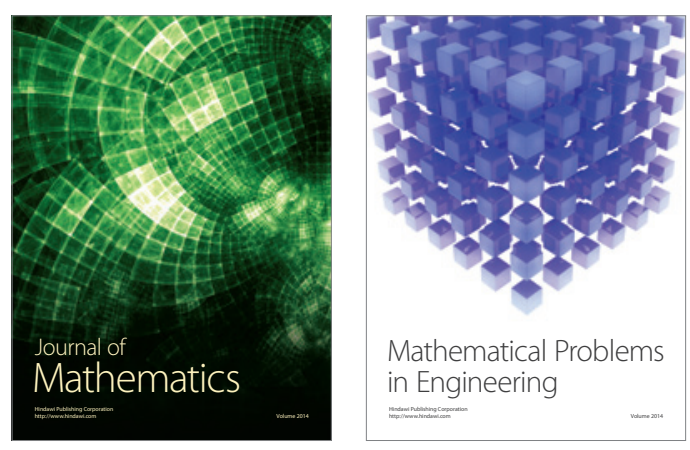

Mathematical Problems in Engineering
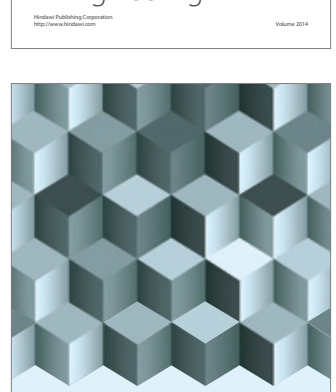

Journal of

Function Spaces
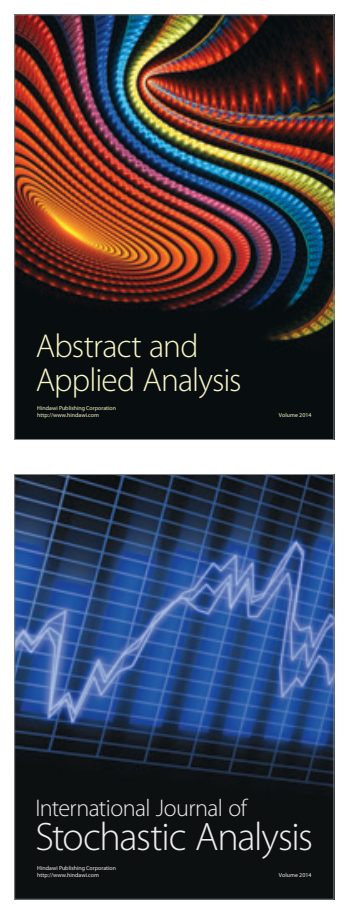

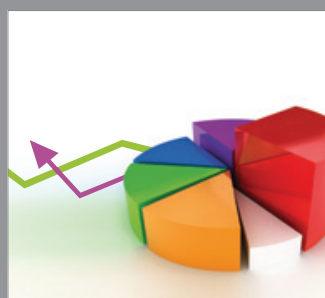

ournal of

Probability and Statistics

Promensencen
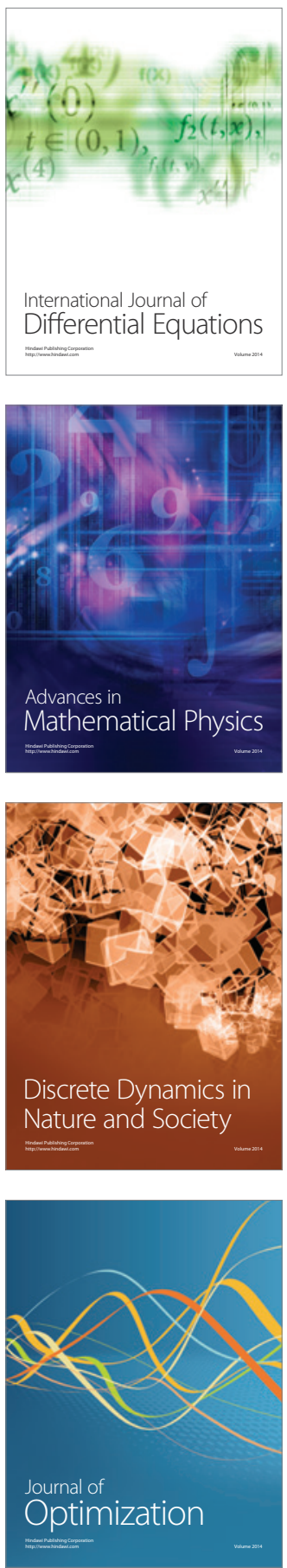